Article

\title{
A Novel Three-Dimensional Non-Destructive Beam-Monitoring Detector
}

\author{
Carolina Belver-Aguilar ${ }^{1}$, Saverio Braccini ${ }^{1, *}$ (D) Tommaso Stefano Carzaniga ${ }^{1}(\mathbb{D}$, \\ Andreas Gsponer ${ }^{1}$, Philipp Daniel Häffner ${ }^{1}$, Paola Scampoli ${ }^{1,2}$ and Matthias Schmid ${ }^{1}$ \\ 1 Albert Einstein Center for Fundamental Physics (AEC), Laboratory for High Energy Physics (LHEP), \\ University of Bern, Sidlerstrasse 5, 3012 Bern, Switzerland; carolina.belver@lhep.unibe.ch (C.B.-A.); \\ tommaso.carzaniga@lhep.unibe.ch (T.S.C.); andreas.gsponer@lhep.unibe.ch (A.G.); \\ philipp.haeffner@lhep.unibe.ch (P.D.H.); paola.scampoli@lhep.unibe.ch (P.S.); \\ matthias.schmid@lhep.unibe.ch (M.S.) \\ 2 Department of Physics "Ettore Pancini", University of Napoli Federico II, \\ Complesso Universitario di Monte S. Angelo, 80126 Napoli, Italy \\ * Correspondence: saverio.braccini@lhep.unibe.ch
}

Received: 20 October 2020; Accepted: 18 November 2020; Published: 20 November 2020

\begin{abstract}
A novel three-dimensional non-destructive beam monitor named $\pi^{3}$ was conceived, realized and tested. It is based on a thin aluminum foil coated with P47 scintillating material mounted on a support, together with a miniaturized CCD camera, both moving along the beam axis. This detector allows reconstructing of the beam distribution along the beam path, providing either an on-line video or a graphical reconstruction of the beam envelope in 3D. The $\pi^{3}$ detector is a general-purpose instrument suitable for any ion accelerator facility. As it is constructed with non-magnetic materials, it can be used to investigate the behavior of the beam inside beam optics components such as magnets. In this paper, we report the development of the first prototype of the $\pi^{3}$ detector, its associated software and the results of the beam tests performed at the Bern medical cyclotron laboratory.
\end{abstract}

Keywords: 3D beam monitor; beam envelope; particle detector; particle accelerator; cyclotron

\section{Introduction}

Beam-monitoring detectors play a crucial role in both commissioning and operations of particle accelerators [1]. They allow the measurement and control of several beam parameters such as beam current, position, size, and shape. They are usually located at fixed positions along the beam path. Destructive detectors are the most common, leading to the impossibility of monitoring and using the beam at the same time. Non-destructive beam monitors are more challenging [2]. Both kinds of devices often feature a graphical output providing mono-dimensional projections or 2-dimensional images of the beam. Several options are available and a remarkable progress has been done in the recent years [3], in particular in the field medical applications [4] as proton-therapy [5]. Cyclotrons for the production of medical radioisotopes are presently common in hospitals and are usually equipped with minimal beam diagnostics devices. This is enough for routine production but specific detectors are needed for novel scientific and clinical developments.

Along this line, at the medical cyclotron in operation at the Bern University Hospital (Inselspital) [6], three different beam monitors have been developed so far by the Laboratory for High Energy Physics (LHEP) of the University of Bern, which are regularly used for multidisciplinary research. The first one is a Faraday cup to measure proton beams down to the pA range [7]. The second one is named UniBEaM and measures the vertical and horizontal beam profiles by means of moving scintillating fibers [8]. This device is commercialized by the Canadian company D-Pace [9]. The third one is named 
$\pi^{2}$, it is based on a fixed scintillating foil and a camera, and allows imaging of the beam spot in 2D and calculation of the beam position and size [10].

This paper describes the development of a novel beam-monitoring detector, named $\pi^{3}$, which represents an evolution of the $\pi^{2}$. The main difference is that in the $\pi^{3}$ both the scintillating foil and the camera are mounted on a moving support located inside the beam pipe in vacuum, allowing assessment of the evolution of the beam envelope along the beam path.

The first prototype of the $\pi^{3}$ has been designed and constructed in the framework of the commissioning of a new irradiation system for the production of non-standard medical radioisotopes using solid target stations [11]. In particular, it was used to study a novel compact magnet embedding two focusing quadruples and two steering magnets into a single device. This detector represents a step forward towards 3D imaging for beam diagnostics. In the following sections, the conceptual design, the experimental setup and the results of the first beam tests are presented and discussed.

\section{Materials and Methods}

The $\pi^{3}$ detector was developed to be part of the instrumentation for the Bern medical cyclotron laboratory. This facility is based on an IBA $18 / 18$ HC cyclotron that provides proton beams with a nominal energy of $18 \mathrm{MeV}$, and beam currents in the range from a few pA to $150 \mu \mathrm{A}$. It is used for the routine production of ${ }^{18} \mathrm{~F}$ for Positron Emission Tomography (PET) and for multidisciplinary research. These two activities can be both efficiently performed thanks to a $6 \mathrm{~m}$ long Beam Transfer Line (BTL), which transports the beam into a separate bunker with an independent access [12], where the experiments described in this paper were conducted. The cyclotron features a Radio Frequency (RF) at $42 \mathrm{MHz}$, which corresponds to the second harmonic of the proton cyclotron frequency. The effects of the RF are not longitudinally visible, and the beam can be considered to be continuous. Transverse beam dynamics was previously extensively studied with the BTL, as reported in Ref. [13].

In the framework of a research program focused on the production of non-standard medical radioisotopes by means of solid targets [11], a novel active irradiation system is under development. It aims at focusing the proton beam produced by a medical cyclotron $(\sim 12 \mathrm{~mm}$ FWHM) into a target of $6 \mathrm{~mm}$ width, and to keep it in position thanks to the feedback given by a specific beam monitor. To be suitable for any medical cyclotron, this system must be compact (about $1 \mathrm{~m}$ long) to fit in existing bunkers of standard dimensions. For this reason, we chose a novel kind of magnet embedding two quadrupoles and two ( $\mathrm{X}$ and $\mathrm{Y}$ ) dipoles within the same device. This apparatus was provided by the company D-Pace (Canada) and is called Mini-PET BeamLine (MBL) [14]. As shown in Figure 1, the magnet is $40 \mathrm{~cm}$ long and weighs $54 \mathrm{~kg}$. The beam pipe features a peculiar shape to minimize the distance of the poles from the beam axis, while ensuring an optimal transmission considering vertical and horizontal focusing and defocusing.
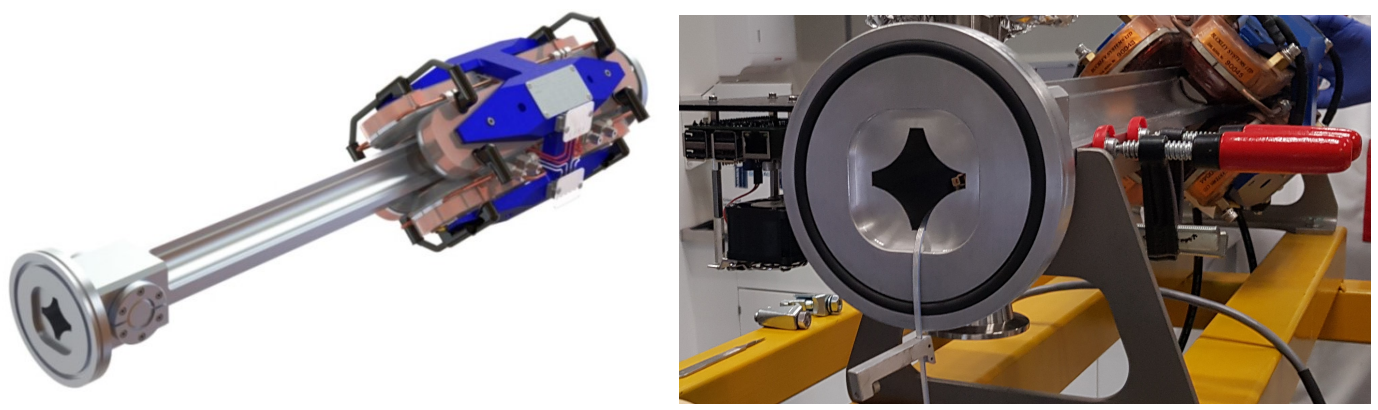

Figure 1. The compact $40 \mathrm{~cm}$ long magnet of the Mini-PET BeamLine (MBL) (left) and the peculiar transverse shape of the beam pipe (right).

The $\pi^{3}$ detector has been designed to study the effects of the MBL on the evolution of the beam along its path. The scheme of the $\pi^{3}$ is shown in Figure 2 (left), where its components are highlighted. A moving support Figure 2 (right) located inside the beam pipe holds the $23.8 \mathrm{~mm}$ diameter circular 
scintillating screen and the miniaturized camera. It is moved along the beam direction by a $660 \mathrm{~mm}$ long screw which is connected to a stepper motor by means of a vacuum feed-through. The screw lead is $1 \mathrm{~mm}$ per revolution and the stepper motor can perform very small steps down to $1 / 100$ rotations. The screw is maintained parallel to the beam axis by means of specific supports (in yellow in Figure 2, left). The moving support holds the P47 aluminum coated foil on one side and a miniaturized CCD camera on the other, as shown in Figure 2 (right). The $\mathrm{P} 47$ scintillating material $\left(\mathrm{Y}_{2} \mathrm{SiO}_{5}\right.$ doped with $\mathrm{Ce}^{+3}$ ) was chosen because it is conductive and has a good light yield with maximum emission around $400 \mathrm{~nm}$ (blue) and a decay time of about $70 \mathrm{~ns}$. The aluminum foil and the P47 coating are both about $1 \mu \mathrm{m}$ thick, producing an energy loss of about $13 \mathrm{keV}$ for the $18 \mathrm{MeV}$ proton beam. The fixed distance between the screen and the camera (Figure 3) allows for an easier reconstruction of the beam image. The CCD used for the first prototype is a KKmoon USB Endoscope Inspection Camera, with a diameter of $5 \mathrm{~mm}$. The resolution of this camera is $640 \times 480$ pixels and it can record images at 8 frames per second. It features an automatic focusing and white balance. This kind of endoscopic camera was chosen for its dimensions and low cost. Indeed, during operations, radiation damage by protons and neutrons deteriorates the performance of the camera, which can however be easily changed and considered to be a consumable. The camera is connected to a Raspberry Pi computer, used for data acquisition, by means of a USB cable under vacuum and a vacuum feed-through. To avoid the cable to interfere with the beam during movement, a pulley system was realized using a weight inside a vacuum pipe mounted vertically and perpendicularly with respect to the beam axis (see Figure 2, left, number 3). A thermal gap filler pad between the camera and the support allows for an optimal heat conduction and prevents overheating the CCD electronics which causes image degradation. As shown in (Figure 2, right), the full volume behind the camera was filled to optimize thermal conduction. For the beam tests of this first prototype, the beam line was terminated using an aluminum beam dump.
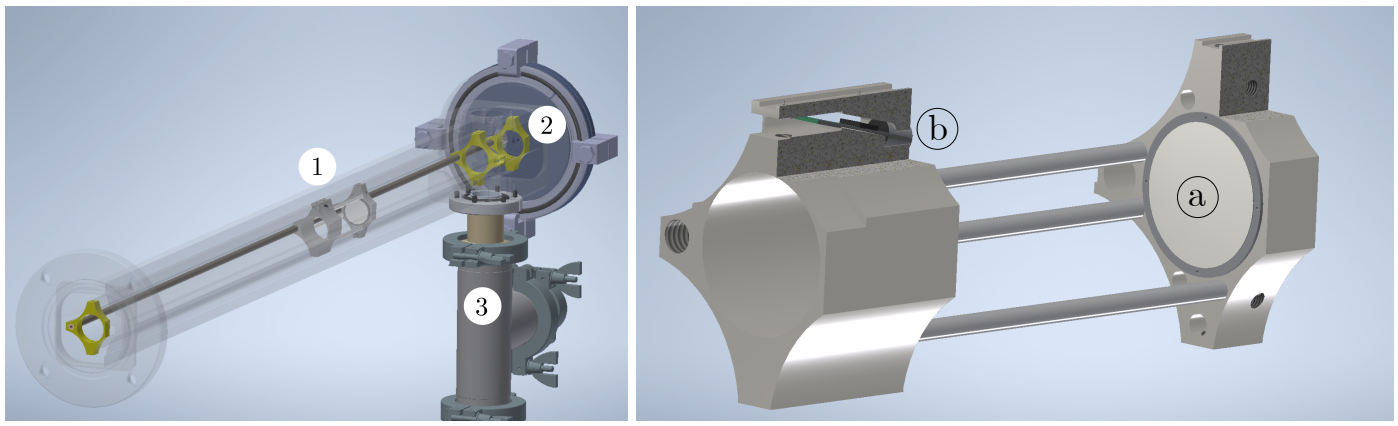

Figure 2. 3D view of the $\pi^{3}$ detector mounted inside of the MBL beampipe (left). The moving support (1), the position of the motor behind the vacuum feed-through (2) and the tube for the pulley system (3) are visible. The support holds the camera that looks towards the P47 coated aluminum foil. The beam comes from the left side. The magnet system of the MBL is not shown. Details of the moving support (right) featuring the scintillating foil (a) and the CCD (b).

The analysis of the images was performed using a Python software specifically developed for the $\pi^{3}$ detector. It performs a pre-processing of the beam images to assess the beam size and position along the MBL [15]. Although the $\pi^{3}$ detector is moving along the MBL, images of the scintillating screen are recorded at regular time intervals. To analyze the images, 2D-Gaussian distributions are fitted to each recorded 2D image. From the 2D-Gaussians, the rotation angle of the beam ellipse is calculated to obtain two projections along the two principal axes. The $x$ and $y$ projections are then fitted by means of a one-dimensional generalized Gaussian curve that, for $x$, is given by the following equation:

$$
f(x)=A_{x} \cdot \exp -\left[\frac{\left(x-\mu_{x}\right)^{2}}{2 \sigma_{x}^{2}}\right]^{p_{x}}
$$


where $\mu_{x}$ gives the centroid of the beam, $\sigma_{x}$ the standard deviation and $p_{x}$ is a fit parameter describing the flatness of the profile. The same function was used for $y$. The use of this function was due to the non-pure Gaussian nature of the beam. The image pre-processing includes some necessary corrections such as those related to the angle between the axis of the camera and that one perpendicular to the scintillating foil (Figure 3) and to convert the image to gray-scale. Finally, a circular mask is applied to the image to remove those parts which are not on the sensitive area of the scintillating screen.

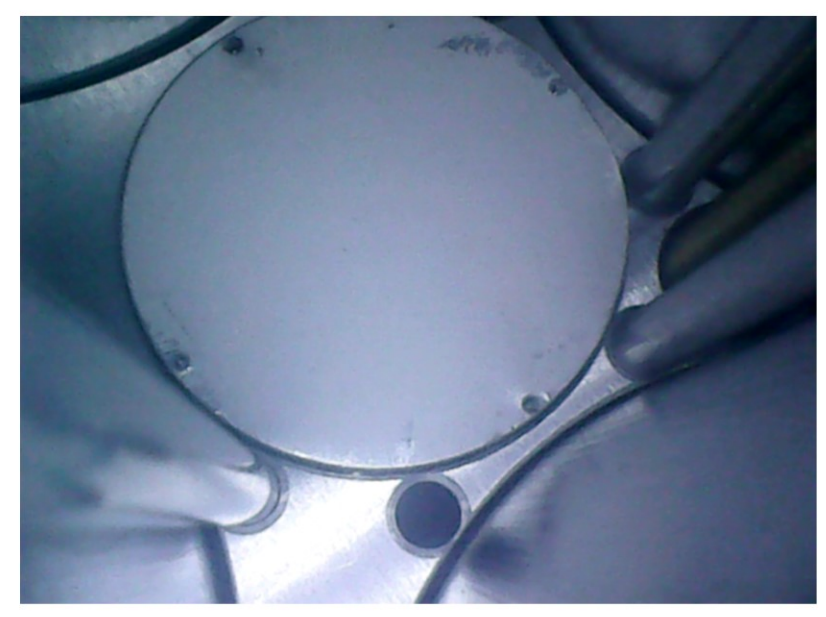

Figure 3. Circular scintillating screen ( $23.8 \mathrm{~mm}$ diameter, total thickness about $2 \mu \mathrm{m})$ illuminated by the LED of the endoscope camera. The perspective angle is corrected by the pre-processing software.

Laboratory tests were first carried out to evaluate the spatial resolution of the detector by using a laser beam. Results showed a high spatial accuracy of $0.3 \mathrm{~mm}$ in both the horizontal and vertical planes. In addition, conversion factors of $0.05 \mathrm{~mm} /$ pixel and $0.07 \mathrm{~mm} /$ pixel were estimated for the horizontal and vertical axis, respectively [15].

After laboratory tests, beam tests were performed using the BTL, as shown in Figure 4. To control the beam position and shape at the entrance of the MBL, the $\pi^{2}$ detector was used. The $\pi^{3}$ detector was mounted inside the beam pipe of the MBL. A system of vacuum valves was used to limit the air flow during pumping to avoid damage to the aluminum coated foil.

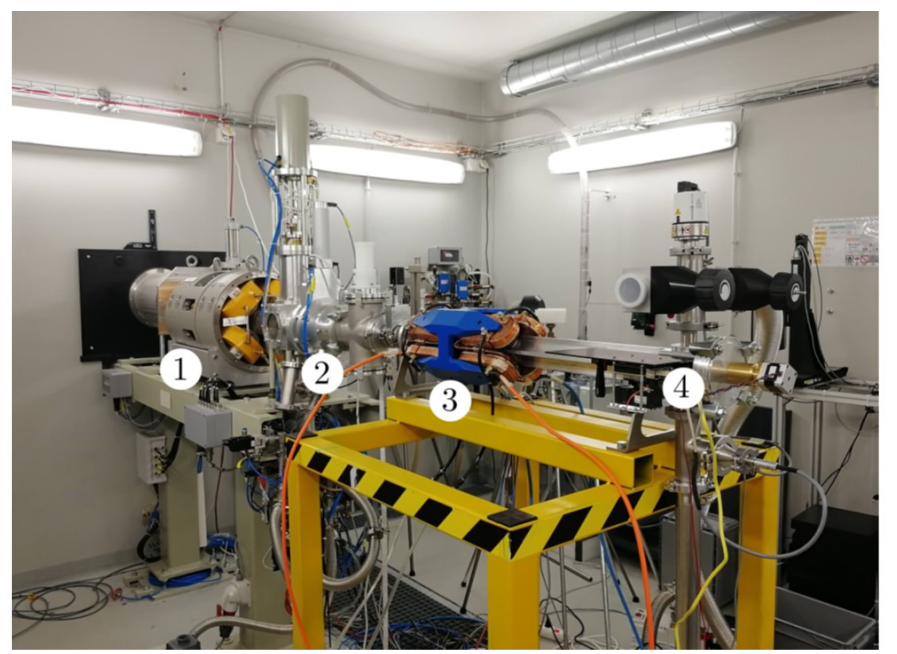

Figure 4. The setup used for the beam tests at the BTL of the Bern medical cyclotron. One of the two BTL quadrupole doublets (1), the $\pi^{2}$ detector (2), the MBL with its beam pipe containing the $\pi^{3}$ detector (3), and the motor with the pulley and vacuum valve system (4) are visible. 


\section{Results of the Beam Tests}

Measurements with the $\pi^{3}$ detector were performed with beam currents between $20 \mathrm{nA}$ and $60 \mathrm{nA}$ to avoid image over-exposure. This current range is unusual for a medical cyclotron designed for radioisotope production and can be obtained by using a specific method developed by our group [7]. Images acquired with $\pi^{3}$ are shown in Figure 5. The image taken at $360 \mathrm{nA}$ (Figure 5, left) shows a clear saturation effect. At low currents, the image can be affected by inhomogeneities of the thickness of the P47 coating, as shown in Figure 5 (right) taken with an early prototype. For the measurements reported in this manuscript, a foil with very high-quality deposition was used to reduce unwanted effects to a negligible level.
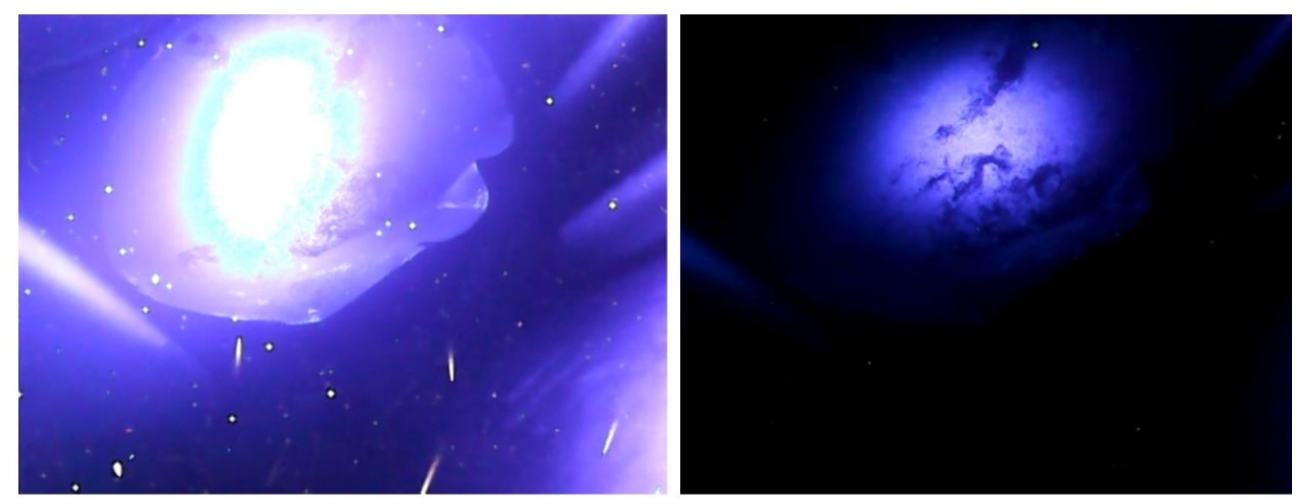

Figure 5. 2D images taken by the $\pi^{3}$ detector with beam currents of $360 \mathrm{nA}$ (left) and $60 \mathrm{nA}$ (right).

For a given beam condition, images were acquired in sequence during the movement of the detector all along the beam path. For the tests presented in this paper, images were acquired at the rate of 8 images/s. For a full pass, a path of $660 \mathrm{~mm}$ was covered in $3 \mathrm{~min}$.

The images were processed using the Python software. As shown in Figure 6, the projections were fitted using a generalized Gaussian curve to calculate the position of the center and the sigma of the beam. The width of the beam and the rotation angle were stored for each image to obtain a full 3D reconstruction of the beam profile.

For these first beam tests, the performance of the $\pi^{3}$ detector was studied using three different beam optics configurations: (1) disabling the MBL magnets (the currents of the two MBL quadrupoles are set to zero), which is equivalent to move the $\pi^{3}$ detector along a drift space; (2) a beam focalized by the BTL entering the MBL (the currents of the two MBL quadrupoles are set to $40 \mathrm{~A}$ ); (3) a flat beam entering the MBL (the currents of the two MBL quadrupoles are set to 0 and $75 \mathrm{~A}$ for the first and second quadrupole, respectively).

The position in $x$ and in $y$ on the beam center is shown in Figure 7 as function of the position along the MBL for the three chosen cases. The error bars correspond to the dominating systematic uncertainty estimated to be of $0.3 \mathrm{~mm}$, mostly due to the accuracy of the mechanics (see laboratory tests described in Section 2). The center of the first quadrupole of the MBL is located at 0 , where the beam enters the MBL, while the center of the second quadrupole is located at $220 \mathrm{~mm}$. Tiny effects are visible, demonstrating the high sensitivity of the $\pi^{3}$ detector. In Figure 7 (top) the drift of the beam is clearly observed. The linear decrease in the $x$ direction is due to a non-perfect alignment of the MBL with respect to the beam path. In Figure 7 (center and bottom), the modest kinks are due to steering effects of the quadrupoles. In fact, the beam was not perfectly centered with respect to the quadrupole axis. It must be noted that some data are missing due to transmission saturation problems between the CCD and the Raspberry Pi computer. 


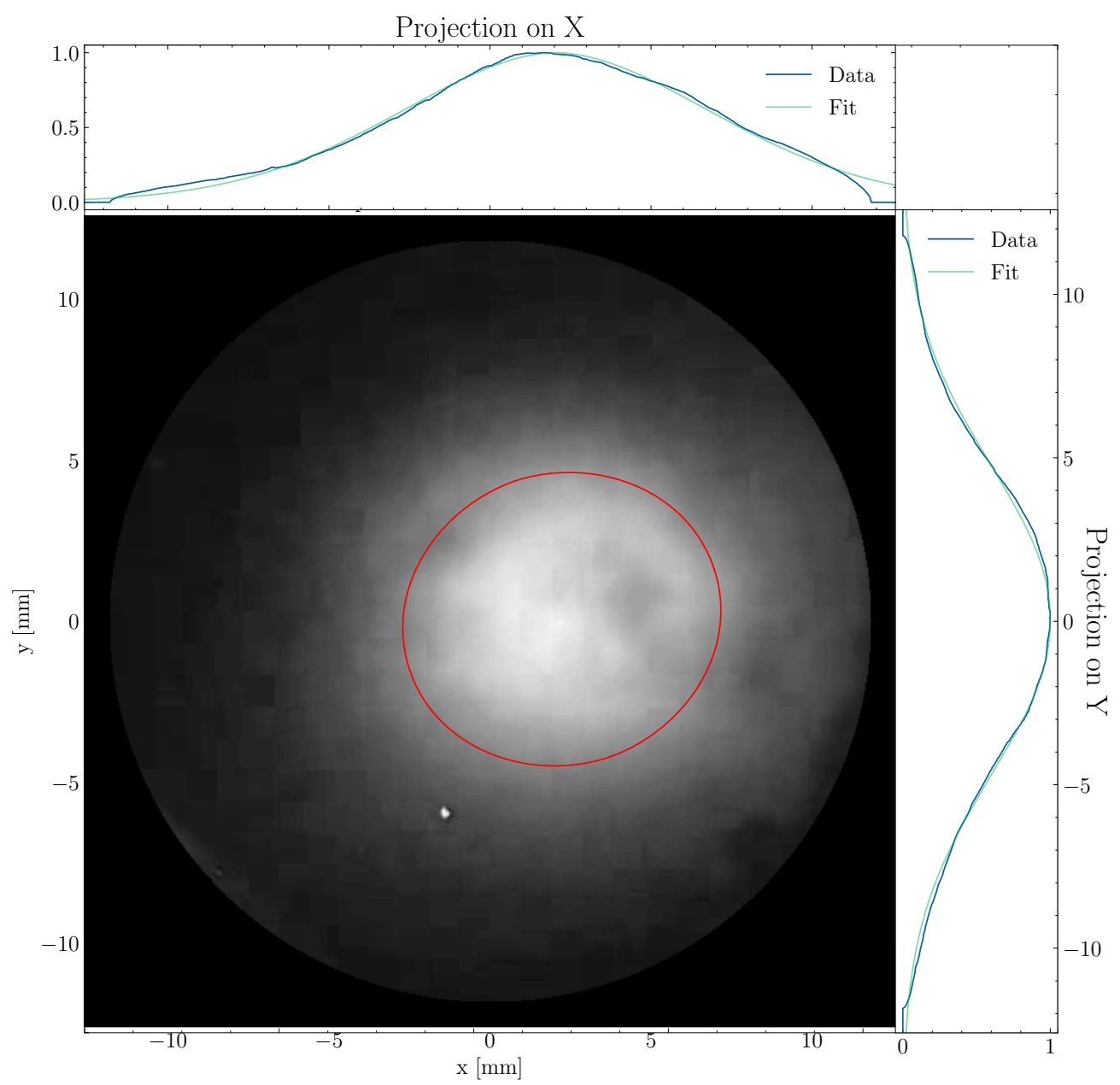

Figure 6. 2D image of the beam, where the ellipse corresponds to the $1 \sigma$ contour. The $x$ and the $y$ projections fitted by a generalized Gaussian curve are shown.

The evolution of the sigma of the beam was studied for the three cases, as shown in Figure 8. The error bars come from the results of the fits. For the first configuration (Figure 8, top), the beam was focused only by the BTL magnets. In this case, the foil and the camera were moved along a drift space, and the sigma increases in both planes as expected. In the second case (Figure 8, center), the beam is focused at the MBL entrance by the BTL magnets and refocused in the MBL. The overall effect results in a small focusing of the beam in the vertical plane, as compared with the drift space, whereas in the horizontal plane it remains basically the same. It must be noted that the focusing power of the BTL is much higher with respect to the one of the MBL. In the third case (Figure 8, bottom), when a flat (not focalized) beam enters the MBL, the MBL is able to reduce the sigma in both planes. The evolution of the values of the fit parameters $\mathrm{p}_{x}$ and $\mathrm{p}_{y}$ is also reported, as shown in Figure 9.

These measurements demonstrate that the $\pi^{3}$ detector can be successfully used to study the evolution of the beam position and shape, highlighting focusing and defocusing effects. 

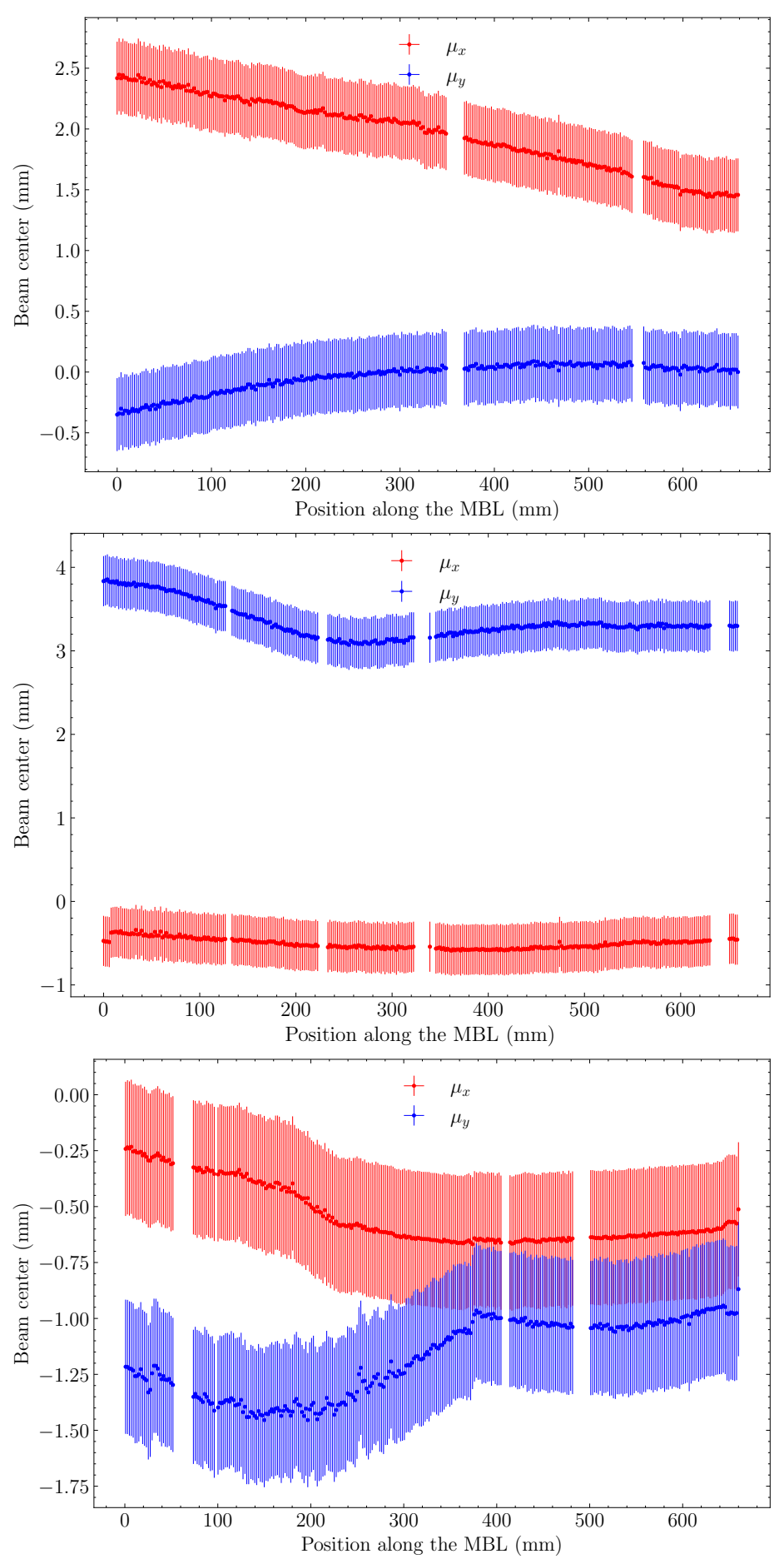

Figure 7. Measured position of the beam centroid in both horizontal (red) and vertical (blue) planes, along the MBL for the three different cases: MBL magnets off (top); a focused beam entering the MBL with magnets on (center); a flat beam entering the MBL (bottom). 

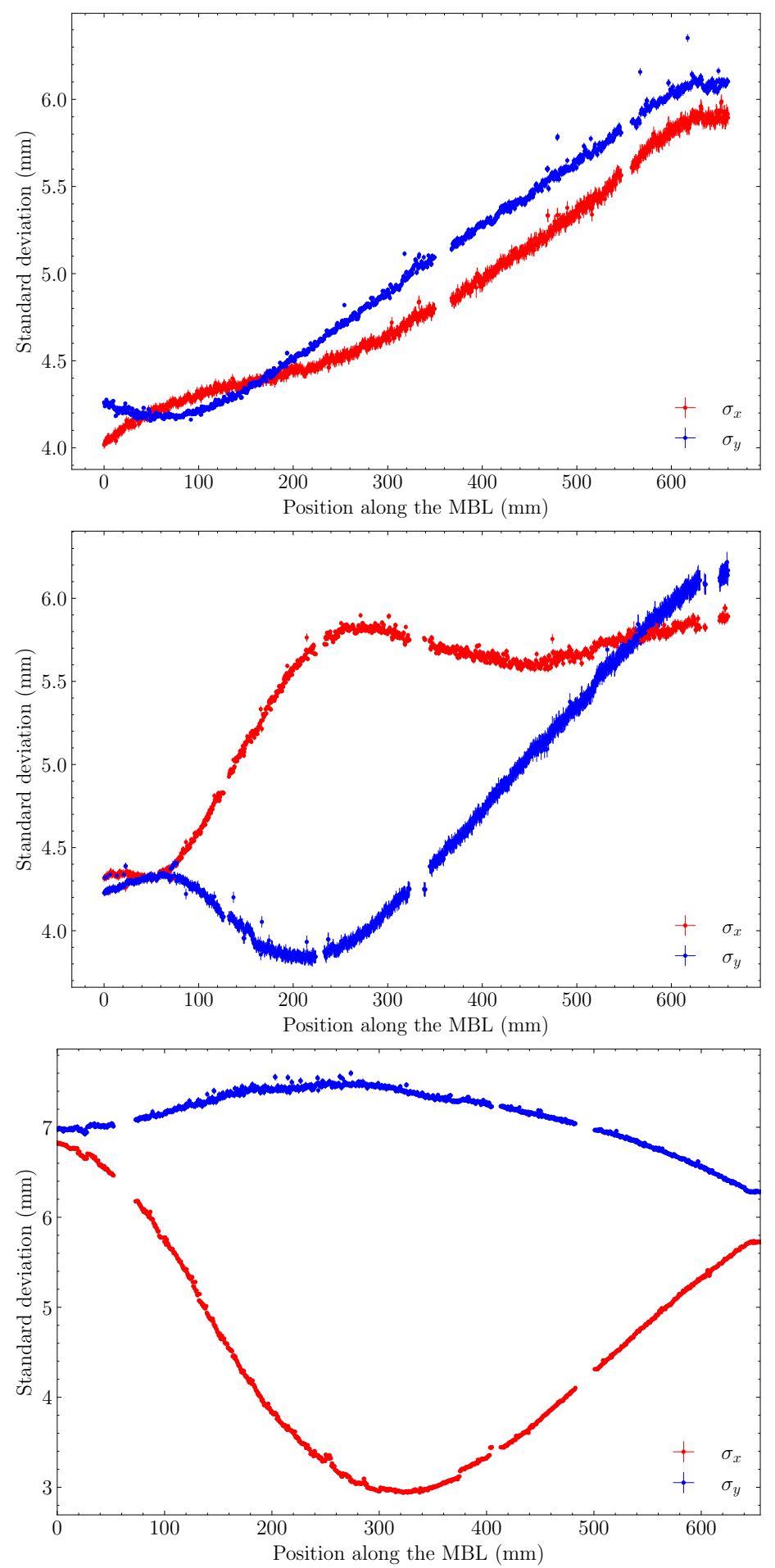

Figure 8. Measured sigma in both horizontal (red) and vertical (blue) planes, along the MBL for the three different cases: MBL magnets off (top); a focused beam entering the MBL with magnets on (center); a flat beam entering the MBL (bottom). 

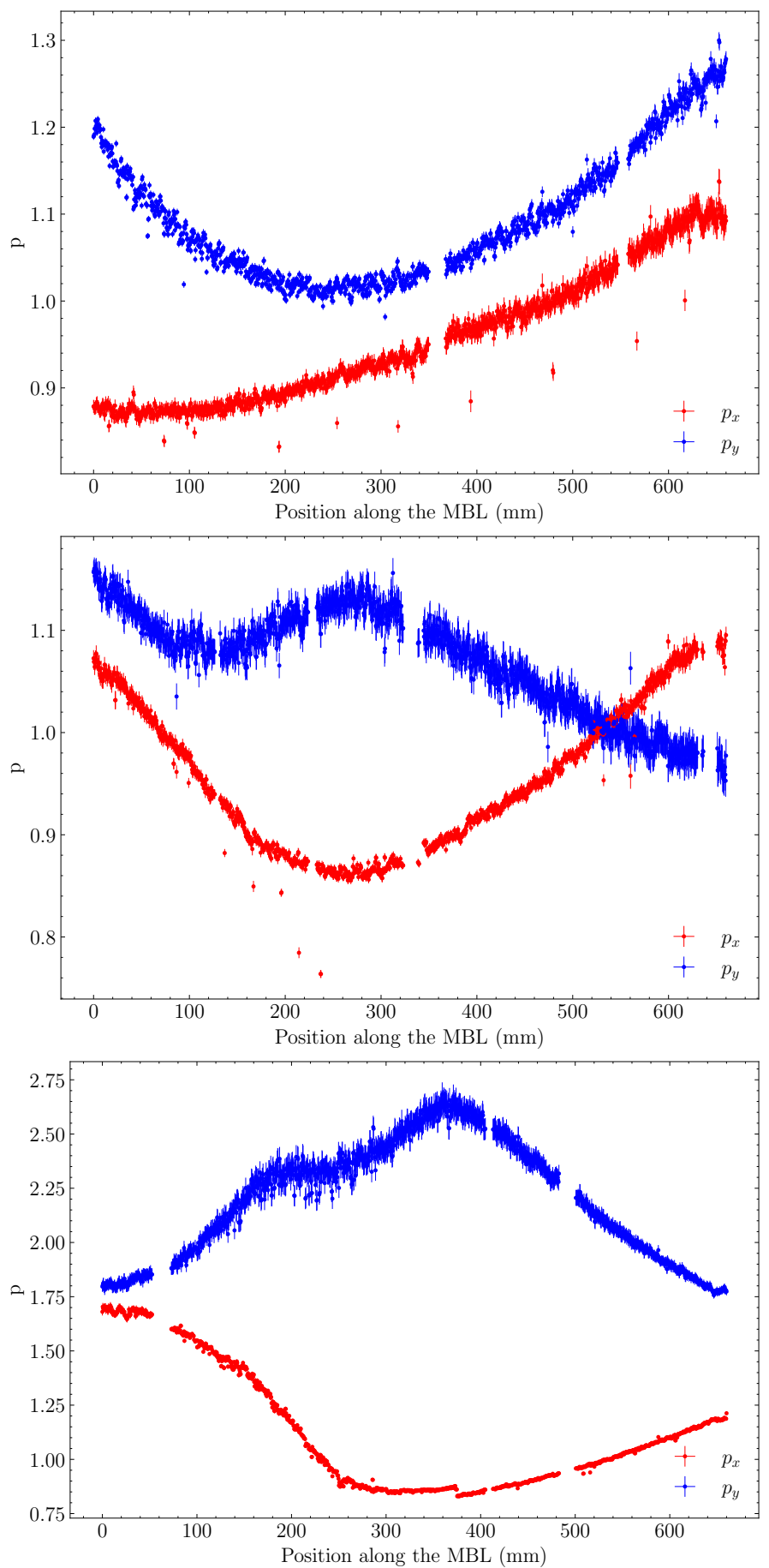

Figure 9. Fitting $\mathrm{p}_{x}$ and $\mathrm{p}_{y}$ parameters in both horizontal (red) and vertical (blue) planes, along the MBL for the three different cases: MBL magnets off (top); a focused beam entering the MBL with magnets on (center); a flat beam entering the MBL (bottom).

Based on the measured parameters along the beam path, the evolution of the sigma of the beam (envelope) can then be fully reconstructed in 3D. Figure 10 shows the evolution of the beam shape for the three cases. For the first configuration (Figure 10, top), the beam envelope increases in both planes due to the drift. In the second configuration (Figure 10, center), the BTL and MBL quadrupoles focus the beam and the effects of the MBL magnets are visible. In the last case (Figure 10, bottom), the MBL can reduce the beam size in both planes. It has to be noted that in this last case and only for Figure 10 (bottom), the parameters $p_{x}$ and $p_{y}$ were set equal to one to avoid graphical artefacts due to the flat beam entering 
the MBL that was larger than the foil of the $\pi^{3}$ detector. A three-dimensional dynamical view of the beam envelope for these three cases is available as videos in the Supplementary Materials.
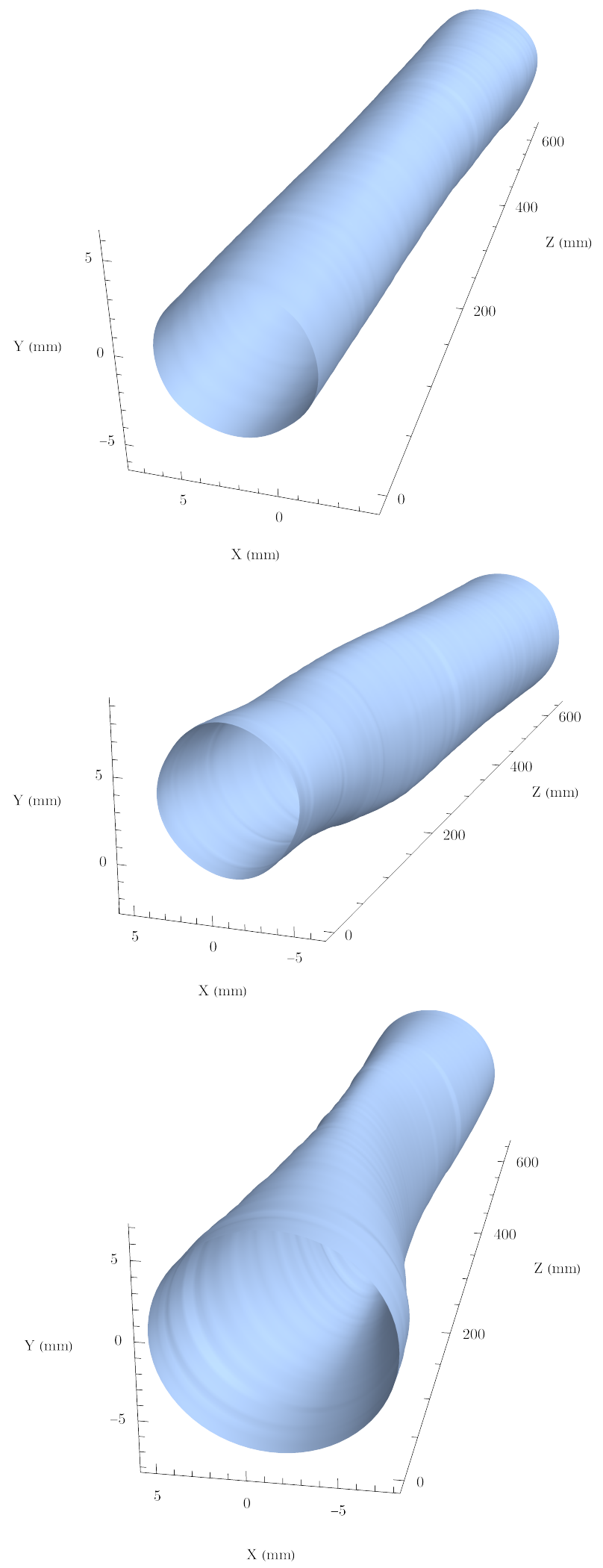

Figure 10. Reconstructed 3D beam envelope, for the three different cases: MBL magnets off (top); a focused beam entering the MBL with magnets on (center); a flat beam entering the MBL (bottom). 


\section{Conclusions and Outlook}

The first prototype of the $\pi^{3}$ detector was designed, built and tested. It is based on a thin aluminum foil coated with P47 scintillating material and a miniaturized CCD camera both moving along the beam axis. It allows the 3D reconstruction of the beam distribution along its path.

Beam tests performed at the Bern medical cyclotron laboratory showed that this detector can reconstruct the behavior of the beam inside a magnet. In particular, it allowed the study of the Mini-PET Beamline, a novel compact magnet that can be used to focus proton beams for the production of non-standard radioisotopes for medical applications. The evolution of the beam along its path was observed either on-line or off-line using a specific post-processing software. Tiny effects due to misalignment or minor steering effects due to quadrupoles in the case of non-optimal centering of the beam could be put in evidence.

The concept at the basis of this instrument is general and can be applied to study any beam line, especially if non-standard beam optics elements are present. We have demonstrated that the $\pi^{3}$ detector can be successfully employed to optimize a novel compact beam line for medical radioisotope production. In proton-therapy cyclotrons, it can be used to characterize therapeutic beams, which feature currents in the nA range.

Based on the promising results obtained with the first prototype of the $\pi^{3}$ detector, further developments are under study. In particular, the use of a camera with programmable ISO and exposure time control will allow the obtaining of a better linearity and increasing the beam current operation range.

Supplementary Materials: The following are available online at http://www.mdpi.com/2076-3417/10/ 22/8217/s1 , Video S1: Fig_10_A_Supp_beam_focused_by_BTL; Video S2: Fig_10_B_Supp_beam_focused _by_BTL_and_MBL; Video S3: Fig_10_C_Supp_flat_beam_focused_by_mbl.

Author Contributions: S.B. and T.S.C. conceived of and designed the $\pi^{3}$ detector; C.B.-A., S.B. and A.G. contributed to the construction of the detector; C.B.-A. and A.G. performed the laboratory tests; C.B.-A., A.G., P.D.H. and M.S. performed the beam tests and analyzed the data; C.B.-A., S.B. and P.S. wrote the paper; C.B.-A., S.B., T.S.C., A.G., P.D.H., P.S., and M.S. revised the paper; S.B. coordinated the project. All authors have read and agreed to the published version of the manuscript.

Funding: This research was partially funded by the Swiss National Science Foundation (SNSF). Grants: 200021_175749, CRSII5_180352,CR23I2_156852.

Acknowledgments: We acknowledge contributions from the LHEP engineering and technical staff, in particular Pascal Lutz, who helped us with the mechanical and electronics challenges during the prototype development and installation.

Conflicts of Interest: The authors declare no conflict of interest.

\section{References}

1. Raich, U. Beam Diagnostics; CAS CERN Accelerator School (CERN-2006-012); Brandt, D., Ed.; European Organization for Nuclear Research (CERN): Geneva, Switzerland, October, 2006.

2. Welsch, C.P. Non-destructive beam profile monitors. In Proceedings of the 8th International Particle Accelerator Conference IPAC 2017, Copenhagen, Denmark, 14-19 May 2017.

3. Proceedings of the 8th International Beam Instrumentation Conference, IBIC 2019, Malmö, Sweden, 8-12 September 2019. Available online: www.jacow.org (accessed on 20 October 2020).

4. Welsch, C.P. Beam diagnostics for medical accelerators. In Proceedings of 5th International Beam InstrumentaAtion Conference, IBIC 2016, Barcelona, Spain, 11-15 September 2016.

5. Klimpki, G.; Psoroulas, S.; Bula, C.; Rechsteiner, U.; Eichin, M.; Weber, D.C.; Lomax, A.; Meer, D. A beam monitoring and validation system for continuous line scanning in proton therapy. Phys. Med. Biol. 2017, 62, 6126-6143. [CrossRef]

6. Braccini, S.; Ereditato, A.; Scampoli, P.; Bremen, K.V. The new Bern cyclotron laboratory for radioisotope production and research. In Proceedings of the IPAC2011, THPS080, San Sebastian, Spain, 4-9 September 2011; p. 3618. 
7. Auger, M.; Braccini, S.; Ereditato, A.; Nesteruk, K.P.; Scampoli, P. Low current performance of the Bern medical cyclotron down to the pA range. Meas. Sci. Technol. 2015, 26, 094006. [CrossRef]

8. Auger, M.; Braccini, S.; Carzaniga, T.S.; Ereditato, A.; Nesteruk, K.P.; Scampoli, P. A detector based on silica fibers for ion beam monitoring in a wide current range. J. Instruments (Jinst) 2016, 11, P03027. [CrossRef]

9. Potkins, D.E.; Braccini, S.; Nesteruk, K.P.; Carzaniga, T.S.; Vedda, A.; Chiodini, N.; Timmermans, J.; Melanson, S.; Dehnel, M.P. A Low-Cost Beam Profiler Based On Cerium-Doped Silica Fibres. Phys. Procedia 2017, 90, 215-222. [CrossRef]

10. Belver-Aguilar, C.; Carzaniga, T.S.; Gsponer, A.; Häffner, P.; Scampoli, P.; Schmid, M.; Braccini, S.; Molinari, G. Development of Novel Non-Destructive 2D and 3D Beam Monitoring Detectors at the Bern Medical Cyclotron. In Proceedings of the IBIC2020, Remote, Brazil, 14-18 September 2020; TUPP32.

11. Braccini, S.; Belver-Aguilar, C.; Carzaniga, T.S.; Dellepiane, G.; Häffner, P.D.; Scampoli, P. Novel Irradiation Methods for Theranostic Radioisotope Production with Solid Targets at the Bern Medical Cyclotron. In Proceedings of the International Conference on Cyclotrons and their Applications (CYC), Cape Town, South Africa, 22-27 September 2019; TUA02.

12. Braccini, S. The new Bern PET cyclotron, its research beam line, and the development of an innovative beam monitor detector. Aip Conf. Proc. 2013, 1525, 144-150.

13. Nesteruk, K.P.; Auger, M.; Braccini, S.; Carzaniga, T.S.; Ereditato, A.; Scampoli, P. A system for online beam emittance measurements and proton beam characterization. J. Instrum. 2018, 13, P01011. [CrossRef]

14. Dehnel, M.P.; Potkins, D.E.; Stewart, T.M. An Integrated Self-Supporting Mini-Beamline for PET Cyclotrons. In Proceedings of the International Conference on Cyclotrons and their Applications (CYC), Vancouver, BC, Canada, 16-20 September 2013; TUPSH014.

15. Gsponer, A. Development and First Test of a Novel Three-Dimensional Beam Monitor. Bachelor's Thesis, University of Bern, Bern, Switzerland, 2019.

Publisher's Note: MDPI stays neutral with regard to jurisdictional claims in published maps and institutional affiliations. 